\title{
DISK REFLECTION SIGNATURES IN THE SPECTRUM OF THE BRIGHT Z-SOURCE GX 340+0*
}

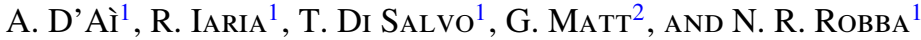 \\ ${ }^{1}$ Dipartimento di Scienze Fisiche ed Astronomiche, Università di Palermo, via Archirafi 36, I-90123 Palermo, Italy; dai@ fisica.unipa.it \\ 2 Dipartimento di Fisica, Università degli Studi Roma Tre, via della Vasca Navale 84, I-00146 Roma, Italy \\ Received 2008 June 26; accepted 2009 January 21; published 2009 February 11
}

\begin{abstract}
We present the preliminary results of a $50 \mathrm{ks}$ long XMM-Newton observation of the bright Z-source GX $340+0$. In this Letter, we focus on the study of a broad asymmetric emission line in the Fe K $\alpha$ energy band, whose shape is clearly resolved and compatible with a relativistically smeared profile arising from reflection on a hot accretion disk extending close to the central accreting neutron star. By combining temporal and spectral analysis, we are able to follow the evolution of the source along its horizontal branch. However, despite a significant change in the continuum emission and luminosity, the line profile does not show any strong correlated variation. This broad line is produced by recombination of highly ionized iron (Fe XXV) at an inferred inner radius close to $13 R_{\mathrm{g}}$, while the fit requires a high value for the outer disk radius. The inclination of the source is extremely well constrained at $35^{\circ}$, while the emissivity index is -2.50 .
\end{abstract}

Key words: line: formation - line: identification - stars: individual (GX 340+0) - X-rays: binaries - X-rays: general

\section{INTRODUCTION}

GX $340+0$ is a bright low-mass X-ray binary (LMXB) belonging to the class of the Z-sources (Hasinger \& van der Klis 1989), and its inferred luminosity is close to the Eddington limit $\left(2 \times 10^{38} \mathrm{erg} \mathrm{s}^{-1}\right.$ for a $1.4 M_{\odot}$ NS $)$. The source has also a radio counterpart, from which a distance of $11 \pm 3 \mathrm{kpc}$ has been estimated (Penninx et al. 1993); radio emission from the counterpart is, however, highly variable, and seems to be correlated with the X-ray flux when the source is in its horizontal branch (HB), but anticorrelated on the other branches (Oosterbroek et al. 1994); it can also show periods of radio quenching, becoming extremely faint (Berendsen et al. 2000).

Temporal analysis studies have shown a complex phenomenology, linked to the accretion state, with characteristics typical of the Z-class (Jonker et al. 2000). The power spectrum shows a low-frequency (tens of $\mathrm{Hz}$ ) quasi-periodic oscillation (QPO) when the source resides on its HB (called HB oscillation, HBO), while at higher frequencies the source shows, at the same time, twin $\mathrm{kHz}$ QPOs whose centroid frequencies are correlated with the HBO peak frequency. Although no burst has yet been observed, the very fast timing variability (at more than $800 \mathrm{~Hz}$ ) and the similarities with the other Z-sources indicate that the compact object is a neutron star.

The spectral properties of the source have not been fully investigated so far; Schulz \& Wijers (1993) studied the 2-12 keV spectrum using EXOSAT data: the spectrum could be well described by a single component due to thermal Comptonization of soft photons, emerging from the NS surface, in a hot corona of moderately optical thickness $(\tau \simeq 5-6)$.

Lavagetto et al. (2004) presented the first broadband $(0.1-200 \mathrm{keV})$ spectrum of the source using data from a BeppoSAX observation. The spectrum could be decomposed into the sum of a soft thermal component of temperature of $0.5 \mathrm{keV}$, an optically thick Comptonized component, and an excess at energies above $20 \mathrm{keV}$ that they fitted with a simple power law. A

\footnotetext{
* Based on observations obtained with XMM-Newton, an ESA science mission with instruments and contributions directly funded by ESA Member States and NASA
}

high-resolution spectrum of the source was studied by Ueda et al. (2005), using a Chandra observation; Chandra data clearly showed the presence of an emission line, fitted with a simple Gaussian profile, at $6.57 \mathrm{keV}$ with $40 \mathrm{eV}$ equivalent width.

In this Letter, we report the results of an XMM-Newton observation of GX 340+0. Thanks to the large collecting area of the EPIC-PN instrument and its good spectral resolution, we find evidence of a strong broad emission line at $\sim 6.7 \mathrm{keV}$; the line profile is very well reproduced by a smeared disk-reflected profile. We find, moreover, evidence of other disk reflection signatures supporting the common scenario, valid for active galactic nuclei (AGN), galactic black hole, and NS systems, that broad iron fluorescence lines are produced by reflection of photons from a hot corona on the surface of an accretion disk that extends very close to the compact object.

\section{OBSERVATION, DATA REDUCTION, AND SPECTRAL SELECTION CRITERIA}

GX 340+0 was observed with XMM-Newton from 2007 September 2 at 13:18:00 to September 3 at 02:32:05 UTC, for a total observing time of 47,640 s (Obs. ID 0505950101). However, because of the high count rate reached in the EPIC-PN CCDs during most of the observation, the effective exposure was reduced by data losses due to telemetry saturation. The total good time intervals thus resulted in 40,562 s effective exposure. In this work we use data collected by the EPIC-PN instrument in fast timing mode; the source region (the background region) has been selected using CCD rows RAWX = 28-48 and RAWY $=0-200$ (RAWX $=2-8$ and RAWY $=$ $0-200)$. No background flaring was present during the observation. We selected only events with PATTERN $\leqslant 4$ (singles and doubles) and FLAG $=0$ and restricted our analysis to the energy range $2.2-11.8 \mathrm{keV}$. We excluded data from PN below $2.2 \mathrm{keV}$ and rely on the Reflection Grating Spectrometers (RGS1 and RGS2, in the 0.4-2.0 keV energy band) for the soft energy band, because PN data present strong systematic residuals of instrumental origin which affect the overall continuum determination. PN data have been progressively rebinned to avoid data oversampling; RGS data are rebinned in 


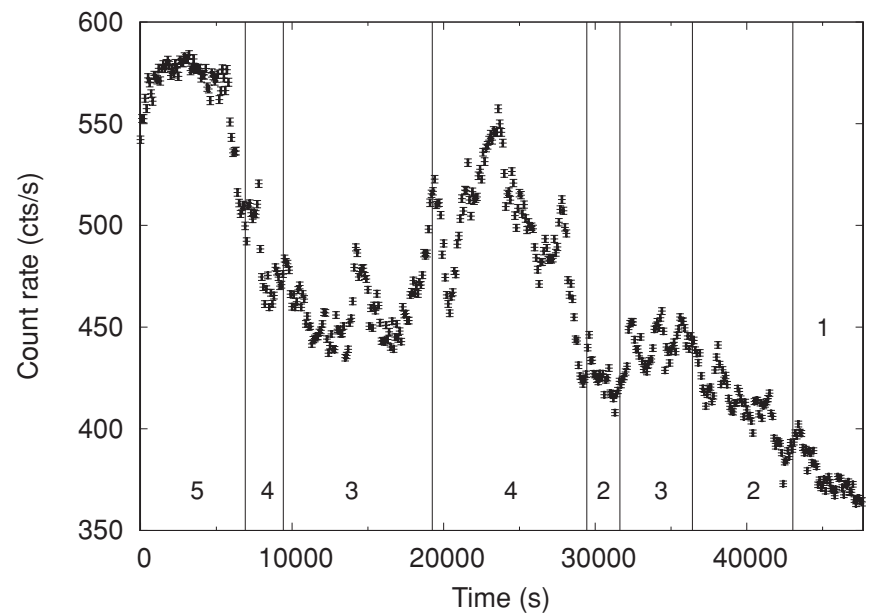

Figure 1. Light curve of GX $340+0$ from the EPIC-PN, starting from MJD 54345.554. Bin time is $100 \mathrm{~s}$. Vertical lines and numbers inside the boxes indicate the time selection for the corresponding energy spectra and the PSD shown in Figure 2.

order to have at least 25 counts per energy channel. Source, background spectra, and response matrices were extracted using the XMM-Newton Science Analysis Software (SAS, ver. 7.1.0). Spectral analysis was performed using Xspec version 12.4.0. Temporal analysis was performed using the FTOOLS version 6.4.1.

In Figure 1, we show the light curve obtained by the EPIC-PN instrument in the $0.5-10 \mathrm{keV}$ energy range. The source shows significant X-ray variability, with a $40 \%$ decrease in count rate between the beginning and end of the observation.

Besides the spectral analysis we also performed a study of the temporal variability. We extracted power spectral densities (PSDs) from the EPIC-PN data in the frequency range $1 / 16 \mathrm{~Hz}$ to $512 \mathrm{~Hz}$. We obtained a single PSD after averaging every 128 PSDs, thus having a total of 20 PSDs for the entire observation. We fitted the averaged PSD using two zero-centered Lorentzians to account for the broadband low-frequency noise, a constant term to fit the white noise, and a Lorentzian to fit a broad QPO. We identify this broad QPO as the HBO (Jonker et al. 2000); this feature, as clearly shown by Jonker et al. (2000) is a good tracer of the overall temporal variability, which in turn, can be used to track the accretion state of the source in the X-ray Color-Color Diagram. With the exception of the first two PSDs, when the source was at the highest count rate, we clearly detect the HBO in all the remaining PSDs. The HBO centroids span a range of frequencies from 17 to $37 \mathrm{~Hz}$, and we noted this frequency to be correlated with the source count rate. We used these results to derive the good time intervals from which we extracted the corresponding energy spectra. After some trials, we decided to slice the observation into five segments; this choice allows us to trail the spectral evolution of the source, still obtaining a high-statistics spectrum to constrain all the spectral parameters. We denote from Spectrum 1 to Spectrum 5 the PN and RGS energy spectra extracted in the time intervals when the HBO frequency was in the 17-22 Hz, 22-27 Hz, 27-32 Hz, $32-37 \mathrm{~Hz}$, and $>37 \mathrm{~Hz}$ (or not detected) range, respectively (see the time-selected intervals shown in Figure 1). In Figure 2, we show the time-averaged power spectra for the time intervals 1-4: the HBO evolved during the observation, moving from lower frequencies, small widths, and high spectral power (Spectrum 1) to higher frequencies, larger widths, and less spectral power (Spectrum 4). The HBO is probably undetected in
Spectrum 5 because of the lower statistics, and because it is too broad to be resolved.

Given the high count rate of the source, we carefully investigated pile-up-related effects by extracting our spectra from rectangular regions with and without the inclusion of the brightest central rows. ${ }^{3}$ Through several different trials, and with the help of the epatplot tool, we concluded that spectra 1-4 are not significantly affected by pile-up, and, as far as we are mostly concerned with the spectral shape of the broad iron Fe $\mathrm{K} \alpha$ line, the spectral parameters are, within the relative uncertainties, self-consistent for region masks with and without the inclusion of the central brightest rows. On the other hand, the time-selected region 5 is affected by pile-up; we extracted, therefore, the associated energy spectrum excluding the central two brightest pixel rows in order to avoid significant pile-up-dependent spectral distortions.

\section{SPECTRAL ANALYSIS}

We analyzed the time-selected spectra independently, adopting a continuum model consisting of soft thermal disk emission (diskbb component; Makishima et al. 1986) and a thermal, harder, blackbody emission (component blackbody). We used for the interstellar absorption the vphabs model with crosssections of Verner et al. (1996) and table abundances of Wilms et al. (2000). We found significant residuals at the neutral iron edge $(7.11 \mathrm{keV})$; leaving the iron abundance free to vary resulted in a significant improvement in the $\chi^{2}$ and in the residuals scattering; iron is under-abundant with respect to the assumed Wilms et al. (2000) solar value (see also Ueda et al. 2005). Superimposed on the continuum emission, a few discrete features are always present: a broad emission feature in the $\mathrm{Fe} \mathrm{K} \alpha$ region, a weaker but also broad emission line at $\sim 3.9 \mathrm{keV}$, and an absorption edge, whose energy threshold is in the $8.7-9.0 \mathrm{keV}$ range.

Although broadband spectra of Z-sources reveal that the hard component is better described by thermal Comptonization models (see, e.g., Lavagetto et al. 2004), XMM-Newton data do not allow us to discriminate among different models of $\mathrm{X}$-ray continuum, or to reliably constrain the parameters of the Comptonization model. We tested, however, using a variety of different spectral models, that the choice of the continuum alters only marginally the overall $\chi^{2}$ value of our fits and the determination of the local feature parameters. In particular, the iron line parameters, which are reported hereafter, are very solid against other choices of continua, with line best-fitting values differing in percentage less than the line relative errors.

We preliminarily investigated the shape of this broad iron line using the total time-averaged PN spectrum (energy range 3-12 keV), excluding only the time interval 5 (see Figure 1) because pile-up affected this part of the observation. Using a Gaussian profile to fit the line emission resulted in a line position of $6.76 \pm 0.02 \mathrm{keV}, 0.24 \pm 0.02 \mathrm{keV}$ width, and $(2.0 \pm 0.2) \times 10^{-3}$ photons $\mathrm{cm}^{-2} \mathrm{~s}^{-1}$ normalization value. Using a relativistically smeared disk-reflected profile (modeled using the diskline profile of Fabian et al. (1989)) resulted in a rest-frame energy of the line at $6.69 \pm 0.02 \mathrm{keV}$, an inner radius $R_{\text {in }}=13 \pm 3 R_{\mathrm{g}}$, an inclination angle $34.6 \pm 1.3$, and emissivity index $-2.50_{-0.13}^{+0.09}$. The $R_{\text {ext }}$ has a best-fitting value $\sim 10^{4} R_{\mathrm{g}}$ but it is rather unconstrained with no upper limit and a $90 \%$ c.l. lower limit at $3000 R_{\mathrm{g}}$. The $\Delta \chi^{2}$ between these two profiles

\footnotetext{
3 Strictly following the SAS thread as described in http://heasarc.nasa.gov/docs/xmm/sas/USG/node63.html.
} 

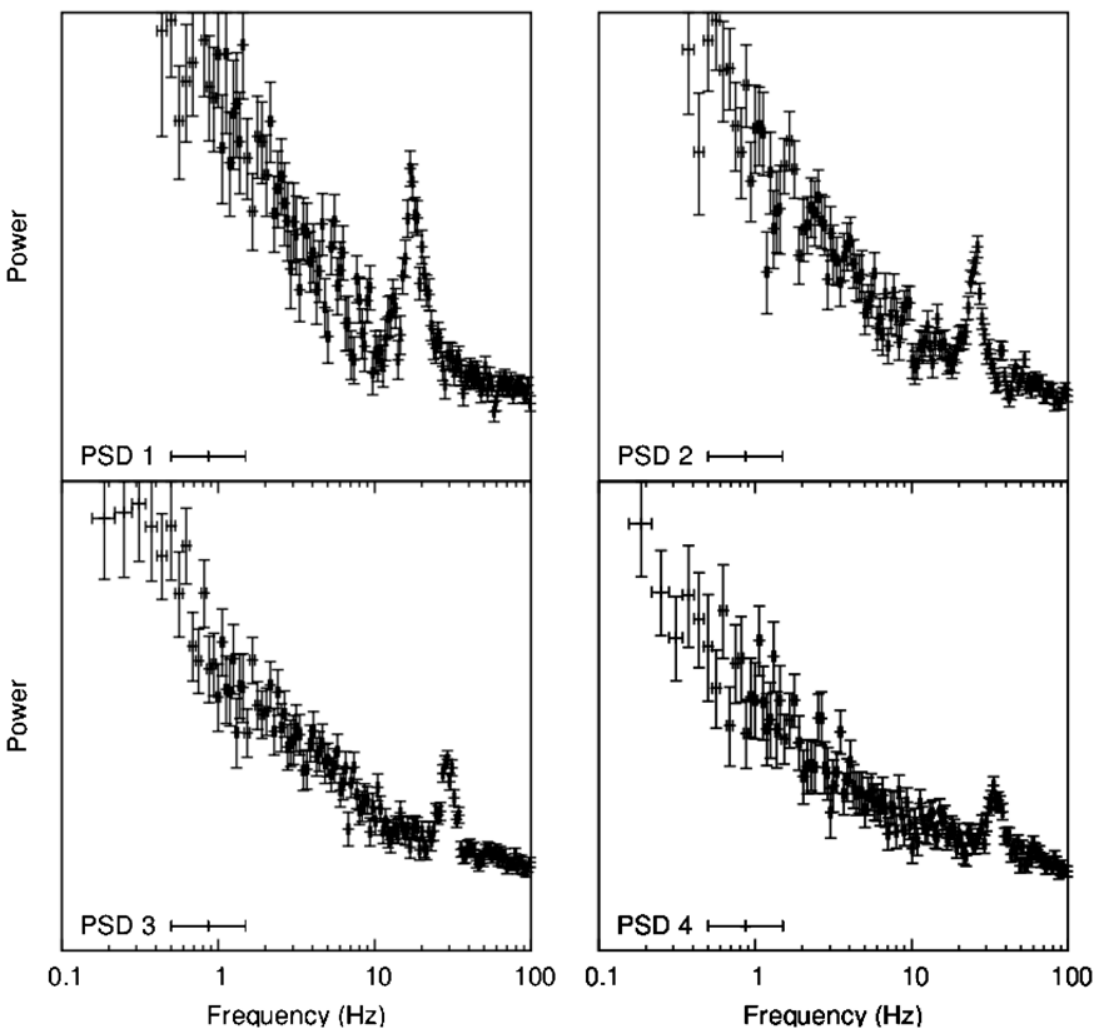

Figure 2. Lehay-normalized power density spectra for four time-selected intervals corresponding to the time selections $1-4$ used in the spectral analysis. Peak frequencies of the HBO are reported in Table 1.

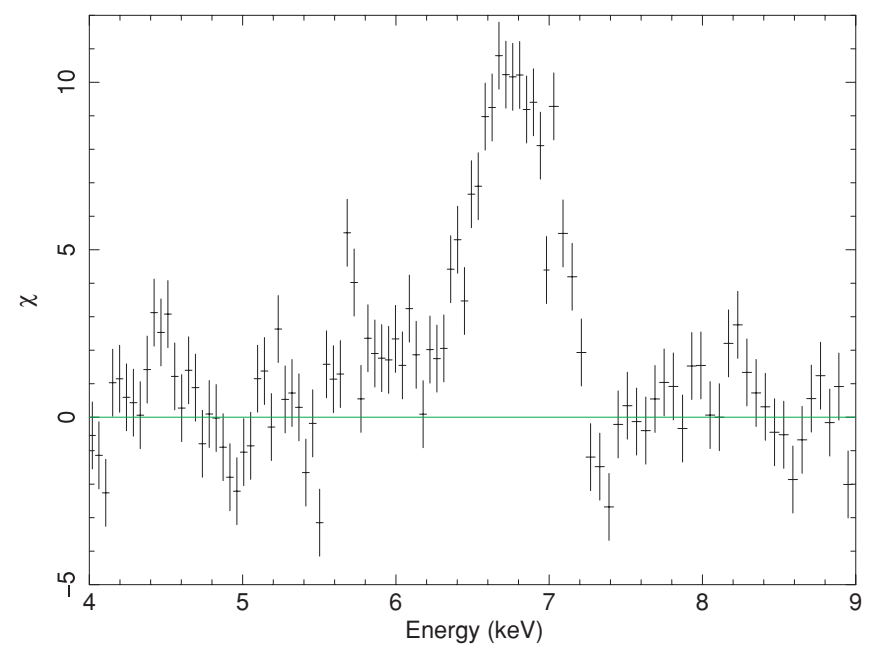

Figure 3. Plot of residuals in terms of sigmas after the subtraction of the iron line emission from the best-fitting model. Data are rebinned for clarity.

is 25 ( +3 degrees of freedom (dof) in the diskline model) indicating the relativistic profile as statistically more favored than the symmetric Gaussian profile. In Figure 3, we show the residuals in terms of sigmas in the iron region, after having set the normalization of the line to zero in the best-fitting model.

We assumed, thereafter, that the $3.9 \mathrm{keV}$ emission line, which we identify with the Ca XIX Ly $\alpha$ transition, is also produced in the disk-reflecting plasma, and we fit it using another diskline component, with $R_{\mathrm{in}},{ }^{4} R_{\mathrm{ext}}$, emissivity index, and inclination bound to the $\mathrm{Fe} \mathrm{K} \alpha$ diskline component.

\footnotetext{
4 Inner and outer disk radii in the diskline model are expressed in units of gravitational radii $\left(R_{\mathrm{g}}\right)$, which for a $1.4 M_{\odot}$ NS corresponds to $2.1 \mathrm{~km}$.
}

We turned, then, to the analysis of the time-selected spectra. Because of the high statistics it is possible to well constrain all the diskline spectral parameters, except the outer radius for which we derived the largest uncertainties. We fixed this parameter to the reference value of $10^{4} R_{\mathrm{g}}$, after having tested that this value lies extremely close $\left(\Delta \chi^{2} \leqslant 1\right)$ to the $\chi^{2}$ minimum for each spectrum. We find that the line shape, within the statistical uncertainties, does not significantly change during most of the observation, despite a significant change of the continuum flux and continuum parameters (see Table 1) with best-fitting values all consistent with the total time-averaged values.

For Spectra 4 and 5, corresponding to the highest luminosities reached by the source during the observation, the temperature of the blackbody component was rather unconstrained by the fit, so that we kept it frozen in the fits to a reference value of $3.5 \mathrm{keV}$, in order to avoid unphysical higher values due to the lack of high energy spectral coverage. In Table 1, we comprehensively show the fitting results for the five time-selected spectra. We representatively show in Figure 4 the data and the residuals in terms of sigmas with respect to the best-fitting model of Spectrum 2.

\section{DISCUSSION AND CONCLUSIONS}

In this Letter, we report on the first unambiguous determination of the broad disk origin of the iron line in the Z-source GX $340+0$. Broad asymmetric iron lines present in the energy range 6.4-6.97 keV have been discovered first in AGN, later in galactic black hole candidates, and very recently also in an NS system (Di Salvo et al. 2005; Bhattacharyya \& Strohmayer 2007; Cackett et al. 2008). The common scenario for the origin of such lines is disk reflection, where the line 
Table 1

Spectral Fitting Results

\begin{tabular}{|c|c|c|c|c|c|}
\hline & Spectrum 1 & Spectrum 2 & Spectrum 3 & Spectrum 4 & Spectrum 5 \\
\hline HBO freq. (Hz) & $17.7 \pm 0.2$ & $25.7 \pm 0.2$ & $29.4 \pm 0.2$ & $34.6 \pm 0.4$ & $37-40^{\mathrm{a}}$ \\
\hline $0.5-2.0 \mathrm{keV}$ flux ${ }^{\mathrm{b}}$ & $-8.588 \pm 0.013$ & $-8.544 \pm 0.008$ & $-8.496 \pm 0.006$ & $-8.456 \pm 0.005$ & $-8.374 \pm 0.005$ \\
\hline $2.0-10.0 \mathrm{keV}$ flux ${ }^{\mathrm{b}}$ & $-8.031 \pm 0.003$ & $-7.992 \pm 0.002$ & $-7.948 \pm 0.002$ & $-7.903 \pm 0.002$ & $-7.835 \pm 0.002$ \\
\hline$N_{\mathrm{H}}^{\mathrm{c}}$ & $9.42_{-0.21}^{+0.26}$ & $9.84 \pm 0.20$ & $9.89 \pm 0.19$ & $10.23 \pm 0.14$ & $10.51 \pm 0.27$ \\
\hline Iron abundance & $0.84_{-0.2}^{+0.13}$ & $0.75 \pm 0.11$ & $0.64 \pm 0.07$ & $0.72 \pm 0.08$ & $0.68 \pm 0.13$ \\
\hline$k T_{\text {disk }}(\mathrm{keV})$ & $1.60_{-0.11}^{+0.23}$ & $1.85 \pm 0.09$ & $1.88 \pm 0.08$ & $2.06_{-0.02}^{+0.03}$ & $2.07 \pm 0.3$ \\
\hline$R_{\mathrm{DBB}}^{\mathrm{d}}(\mathrm{km})$ & $9.7_{-1.7}^{+1.3}$ & $7.8 \pm 0.6$ & $7.6 \pm 0.4$ & $8.0 \pm 0.4$ & $10.5 \pm 0.4$ \\
\hline$k T_{\mathrm{bb}}(\mathrm{keV})$ & $2.55_{-0.12}^{+0.22}$ & $2.89_{-0.20}^{+0.23}$ & $2.98 \pm 0.20$ & $>3.5$ & $>3.5$ \\
\hline Fe $E_{\text {line }}(\mathrm{keV})$ & $6.70 \pm 0.03$ & $6.72 \pm 0.03$ & $6.67 \pm 0.03$ & $6.72 \pm 0.03$ & $6.78_{-0.05}^{+0.06}$ \\
\hline$R_{\text {in }}\left(R_{\mathrm{g}}\right)$ & $16_{-8}^{+20}$ & $13_{-7}^{+5}$ & $14 \pm 2$ & $13_{-7}^{+6}$ & $7_{-1}^{+18}$ \\
\hline$R_{\mathrm{ext}}^{\mathrm{e}}\left(R_{\mathrm{g}}\right)$ & $10^{4}$ & $10^{4}$ & $10^{4}$ & $10^{4}$ & $10^{4}$ \\
\hline Inclination (deg) & $40_{-5}^{+10}$ & $33_{-2.0}^{+2.5}$ & $36.2_{-1.3}^{+0.9}$ & $35.7 \pm 1.7$ & $35^{\mathrm{e}}$ \\
\hline Betor $^{f}$ & $2.38_{-0.10}^{+0.14}$ & $2.46_{-0.07}^{+0.11}$ & $2.52 \pm 0.07$ & $2.47 \pm 0.07$ & $2.50^{\mathrm{e}}$ \\
\hline Fe norm. ${ }^{g}$ & $5.5_{-0.8}^{+0.6}$ & $5.1 \pm 1.0$ & $5.7 \pm 0.5$ & $4.0 \pm 0.6$ & $5.6_{-1.2}^{+3}$ \\
\hline Line equivalent width $(\mathrm{eV})$ & $62_{-10}^{+15^{\circ}}$ & $43_{-7}^{+17}$ & $45 \pm 5$ & $33 \pm 4$ & $29 \pm 10$ \\
\hline $\mathrm{Ca} E_{\text {line }}(\mathrm{keV})$ & $3.87 \pm 0.07$ & $3.93_{-0.10}^{+0.17}$ & $3.94 \pm 0.05$ & $3.94 \pm 0.06$ & $3.92 \pm 0.07$ \\
\hline Ca norm..$^{\mathrm{g}}$ & $0.55 \pm 0.06$ & $0.63 \pm 0.1$ & $1.5 \pm 0.3$ & $1.4_{-0.9}^{+1.9}$ & $3.7_{-1.4}^{+2.7}$ \\
\hline Line equivalent width $(\mathrm{eV})$ & $2.4_{-1.7}^{+2.2}$ & $1.7_{-0.7}^{+1.5}$ & $5.6_{-1.0}^{+1.5}$ & $4.1_{-1.4}^{+1.7}$ & $7_{-4}^{+3}$ \\
\hline Edge $E(\mathrm{keV})$ & $8.85 \pm 0.10$ & $8.97 \pm 0.10$ & $8.94 \pm 0.10$ & $8.80_{-0.07}^{+0.10}$ & $8.91_{-0.09}^{+0.22}$ \\
\hline Edge $\tau\left(\times 10^{-2}\right)$ & $4 \pm 1$ & $3 \pm 1$ & $3 \pm 1$ & $3.0_{-0.7}^{+0.6}$ & $3.7 \pm 1.6$ \\
\hline$\chi_{\text {red }}^{2}($ dof $)$ & $0.974(674)$ & $1.083(771)$ & $1.244(908)$ & $1.186(866)$ & $0.996(766)$ \\
\hline
\end{tabular}

Notes. Best-fitting values and associated errors for spectra $1-5$. Errors quoted at $\Delta \chi^{2}=2.7$.

${ }^{a}$ QPO not resolved in the PSD; value inferred to be in this range from Jonker et al. (2000). See also Figure 2.

b Log to base 10 of the unabsorbed flux in units of erg $\mathrm{cm}^{-2} \mathrm{~s}^{-1}$.

${ }^{\mathrm{c}}$ In units of $10^{22}$ atoms $\mathrm{cm}^{-2}$.

${ }^{\mathrm{d}}$ Inner disk radius in $\mathrm{km}$, as derived from the normalization parameter $N$ of the diskbb component: $R_{\mathrm{DBB}}=D \sqrt{N / \cos (\theta)}$; for the calculation, we assume a distance $(D)$ of $11 \mathrm{kpc}$ and an inclination angle $(\theta)$ of $35^{\circ}$.

e Frozen parameter during the fitting procedure.

${ }^{\mathrm{f}}$ Index of the emissivity power law that scales as $r^{- \text {(Betor) }}$.

$\mathrm{g}$ Normalization values of the diskline component in units of $10^{-3}$ photons $\mathrm{cm}^{-2} \mathrm{~s}^{-1}$.

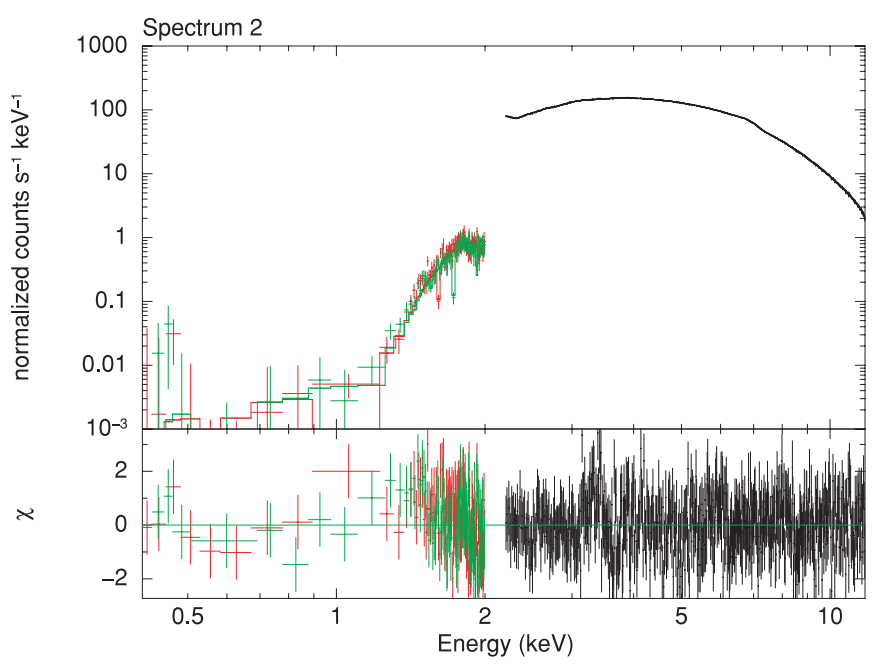

Figure 4. Plot of data (upper panel) and residuals (lower panel) for the representative Spectrum 2.

deformation primarily arises from the high Keplerian velocity fields of the disk-reflecting matter and from general relativistic effects produced by the intense gravitational well of the compact object. Our analysis reveals, for the first time in an NS accreting at the Eddington limit, that the iron line shape in GX $340+0$ is unambiguously described by a disk reflection profile, showing also evidence of other reflection signatures (an absorption edge at $\sim 8.8 \mathrm{keV}$ and a broad Ca XIX emission line).
During this $X M M-N e w t o n$ observation, we can closely follow the spectral evolution of GX 340+0 through all of its HB. At its leftmost point, corresponding to the lowest count rate and to a frequency of the $\mathrm{HBO}$ of $\sim 17 \mathrm{~Hz}$, we derive the lowest temperatures both for the disk $(1.60 \mathrm{keV})$ and the hard emission $(2.55 \mathrm{keV})$; as the source moves toward the hard apex, there is a continuum rising of the temperatures and of the X-ray flux; assuming a distance of $11 \pm 3 \mathrm{kpc}$ and an inclination of the disk as inferred from the diskline profile, we derive a change in the source luminosity from $(1.67 \pm 0.1) \times 10^{38} \mathrm{erg} \mathrm{s}^{-1}$ (Spectrum 1) to $(2.57 \pm 0.2) \times 10^{38} \mathrm{erg} \mathrm{s}^{-1}$ (Spectrum 5). The inner disk radius as derived from the diskbb normalization (see Table 1) gives a value in the $8-11 \mathrm{~km}$ range; considering that the inner disk radius can be underestimated by a factor of two (Merloni et al. 2000), we find that these values are in agreement with the ones derived from the diskline line profile.

Despite this remarkable continuum change, the profile of the broad iron diskline does not sensibly change. The rest-frame energy of the diskline component present in the iron region indicates that the line is most probably produced by He-like iron ions at a short distance from the compact source. In Spectrum 5, because the inclination angle and the emissivity of the line were badly constrained by the fit, we kept them frozen to the best values of the time-averaged spectrum; we noted, therefore, a small shift of the rest-frame line energy toward higher energies, possibly indicating a greater contribution from the H-like ions.

The equivalent width of the line decreases from Spectrum 1 $(\sim 60 \mathrm{eV})$ to Spectrum $5(\sim 30 \mathrm{eV})$, indicating that the line does not respond to the soft X-ray flux, which on the contrary 
increases, but presumably to the hard X-ray emission, above $12 \mathrm{keV}$, which falls out from the observed spectral coverage of the EPIC-PN instrument. The inclination angle of the disk and the emissivity index are very well constrained by our fits, with relative uncertainties of only a few percent. Contrary to what is found in galactic black holes, the emissivity index is not very steep, indicating a more extended illuminating corona above the disk; we are not able to identify any significant change in the value of this parameter during the observation, despite a large increase in the temperatures of the continuum components and their related fluxes.

Recently, Laurent \& Titarchuk (2007) proposed an alternative scenario for the formation of broad iron lines in LMXBs, where extensive red wings could be formed by recoil of line photons in an optically thick medium expanding, or converging, at relativistic velocities. Since a spectral line model, adapted to this scenario, is not yet available for fitting X-ray data, we are not able to test this scenario using our data. However, as pointed out in Pandel et al. (2008) for the case of 4U 1636-536, we note that the lack of a narrow core and the presence of a blue wing in the iron line profile of GX $340+0$ is a strong indication of the disk reflection origin. Moreover, the anticorrelation which is observed between the continuum flux and the line equivalent is difficult to reconcile with this scenario, given that the source of the line photons must also contribute to the continuum emission, either in the form of disk emission or in the blackbody harder component.

Most theoretical models on the QPO generation in NS LMXB agree to identify the shortest dynamical timescales, i.e., the highest frequency QPO at $\sim 1 \mathrm{kHz}$, with the frequency of the Keplerian motion of matter at the inner rim of an accretion disk. From the HBO frequency and the relation known to exist between this and the upper $\mathrm{kHz}$ QPO frequency (Jonker et al. 2000), we can infer the upper $\mathrm{kHz}$ QPO to be in the 550 $750 \mathrm{~Hz}$ frequency range. For a typical $1.4 M_{\odot} \mathrm{NS}$, this frequency range corresponds to an inner disk radius of $(9.8-12.1) R_{\mathrm{g}}$. The diskline inferred inner radii for the time-selected spectra are all fully consistent within this range, and give support to the identification of the highest QPO frequency with the inner disk Keplerian frequency. Our fit results seem also to indicate a decrease in the value of the inner disk radius as the source moves toward higher luminosities. The errors associated with this parameter are, however, still too large to claim a strict correlation, and longer exposures are needed to assert the significance of such a relation.

The spectra show, together with the broad $\mathrm{Fe} \mathrm{K} \alpha$ emission line, other reflection signatures, such as a broad Ca XIX resonance line and a high-energy absorption edge compatible with the Fe xxv K-edge ( $8.83 \mathrm{keV}$ energy in the laboratory frame). The extraordinary good quality of the EPIC-PN spectrum of this source will allow us to test even more recent theoretical line profiles and fully self-consistent disk reflection models. We aim to expand this analysis in a forthcoming paper.

The authors express their gratitude to the EPIC-PN team and, especially, to Matteo Guainazzi for the kind assistance offered to us in the process of data extraction and reduction of the PN data. We would like to thank the anonymous referee for the careful reading of the manuscript and the many suggestions. We also thank Dr. Giuseppe Lavagetto for useful discussions.

\section{REFERENCES}

Berendsen, S. G. H., Fender, R., Kuulkers, E., Heise, J., \& van der Klis, M. 2000, MNRAS, 318, 599

Bhattacharyya, S., \& Strohmayer, T. E. 2007, ApJ, 664, L103

Cackett, E. M., et al. 2008, ApJ, 674, 415

Di Salvo, T., Iaria, R., Méndez, M., Burderi, L., Lavagetto, G., Robba, N. R., Stella, L., \& van der Klis, M. 2005, ApJ, 623, L121

Fabian, A. C., Rees, M. J., Stella, L., \& White, N. E. 1989, MNRAS, 238, 729

Hasinger, G., \& van der Klis, M. 1989, A\&A, 225, 79

Jonker, P. G., et al. 2000, ApJ, 537, 374

Laurent, P., \& Titarchuk, L. 2007, ApJ, 656, 1056

Lavagetto, G., Iaria, R., di Salvo, T., Burderi, L., Robba, N. R., Frontera, F., \& Stella, L. 2004, Nucl. Phys. B: Proc. Suppl., 132, 616

Makishima, K., Maejima, Y., Mitsuda, K., Bradt, H. V., Remillard, R. A., Tuohy, I. R., Hoshi, R., \& Nakagawa, M. 1986, ApJ, 308, 635

Merloni, A., Fabian, A. C., \& Ross, R. R. 2000, MNRAS, 313, 193

Oosterbroek, T., Lewin, W. H. G., van Paradijs, J., van der Klis, M., Penninx, W., \& Dotani, T. 1994, A\&A, 281, 803

Pandel, D., Kaaret, P., \& Corbel, S. 2008, ApJ, 688, 1288

Penninx, W., Zwarthoed, G. A. A., van Paradijs, J., van der Klis, M., Lewin, W. H. G., \& Dotani, T. 1993, A\&A, 267, 92

Schulz, N. S., \& Wijers, R. A. M. J. 1993, A\&A, 273, 123

Ueda, Y., Mitsuda, K., Murakami, H., \& Matsushita, K. 2005, ApJ, 620, 274

Verner, D. A., Ferland, G. J., Korista, K. T., \& Yakovlev, D. G. 1996, ApJ, 465, 487

Wilms, J., Allen, A., \& McCray, R. 2000, ApJ, 542, 914 